\title{
UM ESTUDO SOBRE CASOS CRÍTICOS DE SOLUÇÃO DO FLUXO DE POTÊNCIA ÓTIMO
}

\author{
Katia Campos de Almeida* \\ katia@labspot.ufsc.br \\ *GSP/LABSPOT, Departamento de Engenharia Elétrica \\ Universidade Federal de Santa Catarina (UFSC) \\ 88040-900, Florianópolis, SC, Brasil
}

\begin{abstract}
This paper analyzes critical cases of the optimal power flow (OPF) problem, for which the OPF algorithms usually fail to find a solution. The analysis is based on a parameterized OPF model and on its optimality conditions, which are firstly derived considering the original OPF formulation, with equality and inequality constraints, and subsequently extended to the formulation adopted by the primal-dual interior point methods. An equivalent of the Brazilian Southern Region system is used to illustrate the critical cases.
\end{abstract}

KEYWORDS: Optimal Power Flow, Newton Method, Interior Point Method, Infeasibility, Multiple Solutions.

\section{RESUMO}

Este trabalho tem como objetivo analisar os casos de difícil resolução do problema de fluxo de potência ótimo (FPO). Tal análise é feita a partir de um problema FPO parametrizado e de suas condições de otimalidade, que são primeiramente derivadas supondo a formulação do problema original, contendo restrições de igualdade e desigualdade, e posteriormente particularizadas à formulação usada nos métodos primais-duais de pontos interiores. Um equivalente do Sistema Sul Brasileiro é usado para ilustrar tais casos críticos.

\footnotetext{
Artigo submetido em 10/05/2005

1a. Revisão em 03/11/2005

2a. Revisão em 14/03/2006

Aceito sob recomendação do Editor Associado

Prof. Carlos A. Castro
}

PALAVRAS-ChaVe: Fluxo de Potência Ótimo, Método de Newton, Método de Pontos Interiores, Infactibilidade, Soluções Múltiplas.

\section{INTRODUÇÃO}

O crescente uso de programas computacionais de fluxo de potência ótimo (FPO) não linear em estudos da operação de sistemas de geração/transmissão tem suscitado o desenvolvimento de metodologias para aumentar a robustez dos algoritmos utilizados em tais ferramentas. Devido às diferenças existentes entre o problema do fluxo de carga e do fluxo de potência ótimo, há ainda muita dificuldade para se interpretar os casos onde os algoritmos usados na resolução do FPO não convergem. Infelizmente, como será visto, nem todos casos críticos são de fácil resolução. Entretanto, a diferenciação desses casos críticos é crucial para a interpretação dos resultados obtidos por tais programas.

Diferentes situações levam um algoritmo à não encontrar uma solução para o problema FPO não-linear. A mais óbvia é quando não existe uma solução que respeite o conjunto de restrições de igualdade e desigualdade impostas no problema. Pode acontecer, entretanto, que os algoritmos não encontrem o ótimo mesmo quando este existe. Essas situações ocorrem quando o processo iterativo atinge um ponto onde (i) as condições de otimalidade de primeira ou de segunda ordem não são satisfeitas ou (ii) as condições de factibilidade não são satisfeitas. Para que o processo iterativo prossiga, pode ser necessário fazer uma modificação considerável da estimativa de solução durante o procedimento. Os algorit- 
mos de otimização não-linear não são, em geral, capazes de fazer tal modificação, o que resulta na divergência. Deve-se ter sempre em mente que, a menos que o FPO seja formulado como um modelo convexo de otimização, somente otimalidade e factibilidade local são asseguradas pela maior parte dos algoritmos.

Embora programas FPO que utilizem modelos lineares do sistema de transmissão sejam capazes de reconhecer casos infactíveis do problema, não existe uma forma fácil de fazer o mesmo quando modelos não lineares são usados (Alaç et al., 1990). Os estudos dos casos de difícil resolução do problema FPO não linear foram feitos após o desenvolvimento do programa computacional baseado no método de Newton (Sun et al., 1984). Num trabalho posterior, foram propostas heurísticas para detectar infactibilidade no algoritmo desenvolvido (Sun et al., 1988). Os estudos sobre casos não convergentes foram aprofundados por Monticelli and Liu (1992), que, após analisarem possíveis causas de falha do método de Newton, propuseram modificações na fatoração do Jacobiano das condições de Karush-Kuhn-Tucker (KKT) feita em cada iteração. Mais recentemente, casos de infactibilidade do FPO associados à capacidade de carregamento dos sistemas foram tratados através de metodologias de corte de carga (Granville et al., 1996; Barboza and Salgado, 2001). Além disso, casos não convergentes genéricos do problema FPO foram tratados através de metodologias baseadas na parametrização das restrições do problema (Oliveira et al., 2003; Moyano, 2003). A maioria desses trabalhos não caracteriza formalmente os diversos problemas que podem levar à divergência dos algoritmos FPO adotados.

As causas da não convergência de algoritmos de FPO podem ser determinadas através da análise do comportamento da solução ótima do problema para variações em seus parâmetros. Esses podem caracterizar o custo, a carga, os dados de linha ou os limites físicos e operacionais do sistema. Podese, assim, definir um caso não crítico de FPO como sendo aquele em que uma variação incremental nos parâmetros do problema resulta em uma alteração também incremental da solução ótima. Em tais casos, os algoritmos não encontram usualmente dificuldades para convergência. Por outro lado, um caso crítico de FPO é aquele em que uma pequena variação em um dos parâmetros do modelo leva a condições que resultam na não convergência dos algoritmos usados. Os casos críticos podem ser classificados em quatro tipos se as restrições de igualdade e desigualdade do problema são tratadas diretamente pelo método de Newton (Almeida and Galiana, 1996).

O presente trabalho é baseado em (Almeida, 1998) e constitui uma continuação do estudo apresentado em (Almeida and Galiana, 1996). Seu objetivo é discutir os resultados obtidos nesse último trabalho e estendê-los ao modelo FPO co- mumente usado em programas computacionais baseados em métodos primais-duais de pontos interiores.

O artigo está organizado da seguinte forma. Em primeiro lugar, o problema FPO parametrizado é formulado e são definidos seus casos críticos de solução. Posteriormente, é descrito o modelo do FPO usado em implementações baseadas nos métodos primais-duais de pontos interiores, sendo os casos críticos particularizados para essa formulação. Em seguida, as conseqüências de tais casos críticos são discutidas. São então mostrados exemplos de casos críticos em um sistema realista e, por fim, tiradas algumas conclusões.

\section{ANÁLISE DO PROBLEMA FPO - FOR- MULAÇÃO ORIGINAL}

O problema FPO parametrizado pode ser expresso como

$$
\min f(\mathbf{x}, \varepsilon)
$$

sujeito a

$$
\begin{gathered}
g_{k}(\mathbf{x}, \varepsilon)=0, k \in K, \\
h_{i}(\mathbf{x}, \varepsilon) \leq 0, i \in I,
\end{gathered}
$$

sendo $\mathbf{x} \in \mathbb{R}^{n}$ o vetor de variáveis, $K=1, \ldots, m, m<n$, e $I=1, \ldots, l$. As funções $f, g_{k}, k \in K$ e $h_{i}, i \in I$ são supostas contínuas e com derivadas de, no mínimo segunda ordem, contínuas.

$\mathrm{O}$ parâmetro $\varepsilon$ representa as quantidades usualmente não controláveis presentes na função objetivo e restrições do problema. Cada valor de $\varepsilon$ define um problema FPO específico. $\mathrm{O}$ vetor $\mathrm{x}$ é usualmente composto pelas magnitudes e ângulos das tensões de barra, taps de transformadores de tensão ou defasadores e compensadores shunt variáveis. O critério de otimização pode ser o custo de produção, perdas de transmissão, desvio do perfil de tensão do nominal ou outros índices. A equação (2) representa os balanços de potência ativa e reativa nas barras do sistema e a equação (3) representa os limites em $\mathbf{x}$ ou funcionais.

O modelo paramétrico (1)-(3) é construído de forma que, em $\varepsilon=0$, seja "relaxado"e possua um mínimo trivial e, em $\varepsilon=1$, volte à forma original. Para tanto, à função objetivo é acrescentado um termo linear e outro quadrático em função de $\varepsilon$, e as cargas e os limites operacionais são também modificados através desse parâmetro. Maiores detalhes podem ser obtidos em (Almeida et al., 1994).

\subsection{Condições de Otimalidade}

Os casos críticos de solução do problema (1)-(3) são definidos a partir das condições de otimalidade. Um ponto $\mathbf{x}$ é um ótimo local de (1)-(3) (Luenberger, 1984): 
(i) Se é um ponto regular do conjunto ativo, $A$, o que implica que o Jacobiano

$$
\mathbf{J}(\mathbf{x}, \varepsilon)=\left[\begin{array}{c}
\nabla_{x} \mathbf{g}(\mathbf{x}, \varepsilon) \\
\nabla_{x} \mathbf{h}_{A}(\mathbf{x}, \varepsilon)
\end{array}\right]
$$

possui posto completo, sendo $\mathbf{h}_{A}$ o vetor formado pelas restrições de desigualdade ativas.

(ii) Somente se as condições de Karush-Kuhn-Tucker (KKT) são satisfeitas:

$$
\nabla_{x} f(\mathbf{x}, \varepsilon)+\sum_{k \in K} \lambda_{k} \nabla_{x} g_{k}(\mathbf{x}, \varepsilon)+\sum_{i \in A} \pi_{i} \nabla_{x} h_{i}(\mathbf{x}, \varepsilon)=\mathbf{0},
$$

$$
\begin{array}{r}
g_{k}(\mathbf{x}, \varepsilon)=0, \quad k \in K, \\
h_{i}(\mathbf{x}, \varepsilon)=0, \quad i \in A, \\
h_{i}(\mathbf{x}, \varepsilon)<0, \quad i \notin A, \\
\pi_{i} \geq 0, \quad i \in A .
\end{array}
$$

(iii) Se a matriz Hessiana do Lagrangeano

$$
\mathbf{H}(\mathbf{z}, \varepsilon)=\nabla_{x}^{2} f(\mathbf{x}, \varepsilon)+\sum_{k \in K} \lambda_{k} \nabla_{x}^{2} g_{k}(\mathbf{x}, \varepsilon)+\sum_{i \in A} \pi_{i} \nabla_{x}^{2} h_{i}(\mathbf{x}, \varepsilon)
$$

for definida positiva no espaço nulo do Jacobiano das restrições de igualdade e desigualdades ativas associadas a multiplicadores de Lagrange estritamente positivos, $\mathbf{J}^{+}$.

Na equação (10), $\mathbf{z}=\left[\mathbf{x}^{T}, \boldsymbol{\lambda}^{T}, \boldsymbol{\pi}_{A}^{T}\right]^{T}$, sendo $\pi_{A}$ o vetor formado por $\pi_{i}, i \in A$. A projeção de $\mathbf{H}$ no espaço nulo de $\mathbf{J}^{+}$ é uma matriz denotada $\mathbf{H}_{p}$.

\subsection{Casos Críticos}

A medida que o parâmetro $\varepsilon$ varia, um novo problema FPO, um novo conjunto ativo e novas condições de otimalidade são definidos. Portanto, podemos representar o conjunto ativo como $A(\varepsilon)$. Seja $\mathbf{z}(\varepsilon)$ a solução das condições (i)-(iii) para um dado $\varepsilon$. Um caso não crítico de FPO é, portanto, aquele em que uma pequena variação em $\varepsilon$ leva a uma também pequena variação em $\mathbf{z}$.

A variação de $\varepsilon$ cria uma trajetória de soluções expressa por $\mathbf{z}(\varepsilon)$. Suponha que, em um dado valor de $\varepsilon, \varepsilon^{0}$, o ótimo seja $\mathbf{z}^{0}$. Se, para todo $\varepsilon$ na vizinhança de $\varepsilon^{0}, V\left(\varepsilon^{0}\right)$, existe um ponto $\mathbf{z}$ que satisfaz as condições de otimalidade (i)-(iii) e este $\mathbf{z}$ está num caminho único, contínuo e diferenciável que passa por $\left(\mathbf{z}^{0}, \varepsilon^{0}\right)$, então uma pequena variação em $\varepsilon$ leva a uma também pequena variação em $\mathbf{z}$. A condição do caminho único, contínuo e diferenciável é satisfeita se, em $V\left(\varepsilon^{0}\right)$ (Guddat et al., 1990):
(A) $\mathbf{J}$ possui posto completo.

(B) As restrições de igualdade ativas se mantêm ativas, ou seja,

$$
\pi_{i}(\varepsilon)>0, i \in A(\varepsilon)
$$

(C) As desigualdades inativas não são violadas, ou seja,

$$
h_{i}(\mathbf{x}, \varepsilon)<0, i \notin A(\varepsilon)
$$

(D) A condição suficiente de segunda ordem (iii) é satisfeita.

Caso as condições (A)-(D) sejam satisfeitas, para todo $\varepsilon$ em $V\left(\varepsilon^{0}\right)$ o Jacobiano das condições de KKT (5)-(7),

$$
\mathbf{W}(\mathbf{z}, \varepsilon)=\left[\begin{array}{cc}
\mathbf{H}(\mathbf{z}, \varepsilon) & \mathbf{J}^{T}(\mathbf{x}, \varepsilon) \\
\mathbf{J}(\mathbf{x}, \varepsilon) & \mathbf{0}
\end{array}\right]
$$

é não singular.

A função não linear $\boldsymbol{\rho}(\mathbf{z}, \varepsilon)=\mathbf{0}$ formada pelas equações (5)-(7) conecta os diferentes problemas FPO definidos pela variação do parâmetro. Essa é, portanto, uma função homotopia, cujo Jacobiano é expresso pela equação (13). Deve-se observar que as soluções de $\boldsymbol{\rho}(\mathbf{z}, \varepsilon)=\mathbf{0}$ são, na realidade, trajetórias pois o número de equações desse sistema excede em um o número de incógnitas.

Para um dado conjunto ativo, que garante as desigualdades (8) e (9), estuda-se o comportamento de (5)-(7) para incrementos em $\varepsilon$. Faz-se, para tanto, uma aproximação do sistema (5)-(7) até o termo de primeira ordem. Se tal aproximação é possível - o que ocorre se $\mathbf{W}$ é inversível - localmente $\left(\mathbf{z}^{0}, \varepsilon^{0}\right)$ é um segmento de reta. Estendendo-se esse raciocínio para todo $0 \leq \varepsilon \leq 1$, tem-se a continuidade de $\left(\mathbf{z}^{0}, \varepsilon\right)$ e a garantia que a trajetória de soluções atingirá a solução do problema original em $\varepsilon=1$. Caso a aproximação linear não seja possível, a continuidade pode ainda ser garantida se uma aproximação de ordem superior puder ser feita. Nesse último caso, entretanto, nem sempre a trajetória atingirá $\varepsilon=1$.

Caso, para um incremento em $\varepsilon$, ocorra violações nas condições (B) ou (C), sendo (A) e (D) satisfeitas, tem-se um ponto de quebra, onde $\mathbf{z}(\varepsilon)$ é não diferenciável. A Figura 1 mostra algumas trajetórias de solução do FPO e alguns pontos de quebra observados para variações em $\varepsilon$. Essas são trajetórias de magnitudes de tensão criadas ao se resolver o problema FPO usando o método de Newton com a estratégia do conjunto ativo (Almeida et al., 1994; Almeida and Galiana, 1996). Em $\varepsilon=0$ tem-se as estimativas iniciais das magnitudes de tensão - 1,0pu -, a medida que $\varepsilon$ cresce, o problema é modificado de maneira que, em $\varepsilon=1$, tem-se o problema original, para o qual os valores ótimos das magnitudes de tensão são os pontos definidos pela interseção das trajetórias com o eixo à direita da Figura. Os casos críticos do pro- 


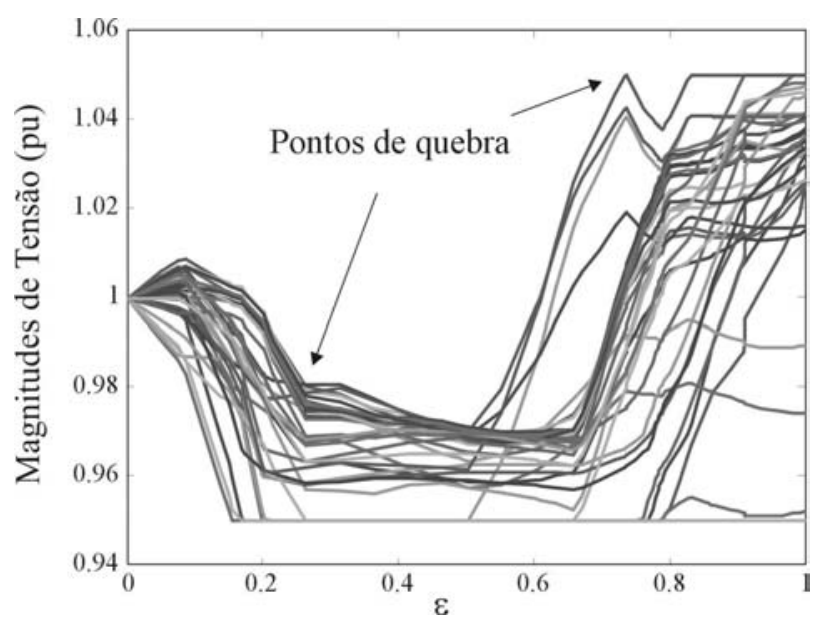

Figura 1: Trajetórias de magnitudes de tensão.

blema (1)-(3) ocorrem quando uma das condições (A)-(D) é violada. A Tabela 1 indica o tipo de violação e as conseqüências de cada caso crítico (Almeida and Galiana, 1996). Os de tipo 1 e 4 ocorrem após modificações no conjunto ativo, enquanto que aqueles de tipo 2 e 3 são definidos apenas pelo incremento no parâmetro.

Tabela 1: Casos Críticos do Problema Original.

\begin{tabular}{|c|c|c|}
\hline Тіро & Violação & Conseqüência \\
\hline 1 & $\begin{array}{l}\text { Violação de (B): Para } \\
\varepsilon \rightarrow \varepsilon^{0} \\
\pi_{q} \rightarrow 0, q \in A\end{array}$ & $\begin{array}{l}\text { Quando a restrição associada a } \pi_{q} \\
\text { é relaxada, há perda de otimalidade: } \\
\text { um autovalor de } \mathbf{H}_{p} \text { se } \\
\text { torna negativo ou nulo. }\end{array}$ \\
\hline 2 & $\begin{array}{l}\text { Violação de (D): Para } \\
\varepsilon \rightarrow \varepsilon^{0} \text {, um autovalor } \\
\text { de } \mathbf{H}_{p} \text { se anula. }\end{array}$ & $\begin{array}{l}\text { Perda de otimalidade. Quando } \\
\varepsilon \rightarrow \varepsilon^{0}, \mathbf{W} \text { se torna } \\
\text { singular. }\end{array}$ \\
\hline 3 & $\begin{array}{l}\text { Violação de }(\mathrm{A}) \text { : Para } \\
\varepsilon \rightarrow \varepsilon^{0}, \mathbf{J} \text { passa } \\
\text { a ter posto incompleto. }\end{array}$ & $\begin{array}{l}\text { Perda de factibilidade. Quando } \\
\varepsilon \rightarrow \varepsilon^{0}, \mathbf{W} \text { se torna singular. } \\
\text { Além disso, pelo menos um dos multi- } \\
\text { plicadores de Lagrange tende a infinito. O } \\
\text { conjunto factível se torna vazio localmente. }\end{array}$ \\
\hline 4 & $\begin{array}{l}\text { Violação de (C): Para } \\
\varepsilon \rightarrow \varepsilon^{0} \\
h_{p} \rightarrow 0, p \notin A .\end{array}$ & $\begin{array}{l}\text { Perda de factibilidade. Se } h_{p} \text { é } \\
\text { introduzida em } A, \mathbf{J} \text { se torna de posto } \\
\text { incompleto. Caso alguma restrição } \\
\text { possa ser relaxada, o processo de } \\
\text { solução continua. Caso contrário, o conjunto } \\
\text { factível se torna vazio localmente. }\end{array}$ \\
\hline
\end{tabular}

Alguns comentários a respeito dos casos críticos devem ser feitos. Em primeiro lugar, deve-se notar que, nos casos onde se indica a perda de otimalidade, supõe-se que essa era garantida nos pontos definidos para $\varepsilon<\varepsilon^{0}$, ou ainda, que as condições (i)-(iii) eram satisfeitas. No caso crítico tipo 1, quando ocorre a liberação de uma restrição anteriormente ativa, $h_{q}$, a otimalidade é perdida porque um dos autovalores de $\mathbf{H}_{p}$ se torna negativo ou nulo. No caso crítico tipo 2, a otimalidade é perdida devido a um dos autovalores de $\mathbf{H}_{p}$ se tornar zero quando há um incremento no parâmetro. O caso crítico tipo 1 também leva à singularidade de $\mathbf{W}$ se um dos autovalores de $\mathbf{H}_{p}$ se anula com a retirada de uma restrição $h_{q}$ do conjunto ativo. Tal retirada diminui em 1 a dimensão de $\mathbf{J}$, aumentando a dimensão de seu espaço nulo e também a de $\mathbf{H}_{p}$. O autovalor associado à nova direção factível, definida pela liberação de $h_{q}$, poderá ser nulo.

A singularidade de $\mathbf{H}_{p}$ pode acontecer em situações mais genéricas: quando as condições de KKT não são satisfeitas, ou seja, $\left(\mathbf{z}^{0}, \varepsilon^{0}\right)$ é uma estimativa de solução do FPO, ou quando, para $\varepsilon<\varepsilon^{0}, \mathbf{H}_{p}$ tem autovalores positivos e negativos, ou ainda, $\mathbf{z}(\varepsilon)$ satisfaz apenas as condições necessárias de otimalidade. Em todas as situações, $\mathbf{W}$ se torna singular.

Os casos críticos tipo 3 e 4 são associados à perda de factibilidade apenas local do problema. Portanto, sua ocorrência não indica necessariamente a inexistência de uma solução para o FPO. Como mostrado na Tabela 1, o caso crítico tipo 4 é definido pela introdução, no conjunto ativo, de uma restrição antagônica àquelas anteriormente ativadas. Para que tal caso seja resolvido, um novo conjunto ativo deve ser encontrado, ou seja, é necessário descobrir quais desigualdades devem ser fixadas nos limites, o que requer a resolução de um problema combinatorial.

Se as restrições de desigualdade do problema são tratadas com auxílio de funções barreira, os casos críticos são aglutinados, conforme visto a seguir.

\section{ANÁLISE DO PROBLEMA MODIFI- CADO}

Na implementação de métodos primais-duais de pontos interiores, o problema (1)-(3) é modificado introduzindo-se variáveis de folga $s_{i}>0, i=1, \ldots, \ell$, nas restrições de desigualdade e acrescentando-se uma função barreira logarítmica à função objetivo para assegurar que essas variáveis sejam estritamente positivas (Fang and Puthempura, 1993). O problema modificado pode ser expresso como

$$
\min f(\mathbf{x}, \varepsilon)-\mu \sum_{i=1}^{\ell} \ln \left(s_{i}\right)
$$

sujeito a

$$
\begin{gathered}
g_{k}(\mathbf{x}, \varepsilon)=0, k \in K, \\
h_{i}(\mathbf{x}, \varepsilon)+s_{i}=0, i \in I,
\end{gathered}
$$

sendo $\mu$ o parâmetro barreira.

O problema (14)-(16) depende de dois parâmetros: $\mu$ e $\varepsilon$. Os casos críticos de solução desse problema são analisados a seguir supondo-se que apenas um desses parâmetros é variado por vez. 


\subsection{Condições de Otimalidade do Pro- blema Modificado}

O ponto solução do problema (14)-(16) deve satisfazer às seguintes condições de primeira ordem:

$$
\begin{gathered}
\nabla_{x} f(\mathbf{x}, \varepsilon)+\sum_{k \in K} \lambda_{k} \nabla_{x} g_{k}(\mathbf{x}, \varepsilon)+\sum_{i \in I} \pi_{i} \nabla_{x} h_{i}(\mathbf{x}, \varepsilon)=\mathbf{0} \\
-\mu s_{i}^{-1}+\pi_{i}=0 \Rightarrow-\mu+s_{i} \pi_{i}=0, i \in I \\
g_{k}(\mathbf{x}, \varepsilon)=0, \quad k \in K \\
h_{i}(\mathbf{x}, \varepsilon)+s_{i}=0, \quad i \in I
\end{gathered}
$$

para $s_{i}>0$ e $\pi_{i} \geq 0, i \in I$.

Além das condições anteriores, o ponto solução deve ser regular e também respeitar a condição suficiente de segunda ordem (iii).

\subsection{Casos Críticos do Problema Modifi- cado}

Os casos críticos do problema modificado podem ser analisados a partir do comportamento das trajetórias de solução da função homotopia $\boldsymbol{\tau}(\mathbf{y}, \mu, \varepsilon)=\mathbf{0}$, formada por (17)-(20) para $\mathbf{y}=\left[\mathbf{x}^{T}, \mathbf{s}^{T}, \boldsymbol{\lambda}^{T}, \boldsymbol{\pi}^{T}\right]^{T}$. A resolução das condições (17)-(20) para $\mu$ ou $\varepsilon$ variável também cria trajetórias de solução. Uma situação de especial interesse é resolução de um problema com $\varepsilon$ fixo através do método primal-dual de pontos interiores. Neste caso, apenas o parâmetro barreira $\mu$ é variado, criando trajetórias de solução. Tal situação é ilustrada pela Figura 2, que mostra o comportamento das magnitudes das tensões durante a resolução do problema FPO modificado. Nela, $\varepsilon$ é fixo enquanto $\mu$ é decrementado. As trajetórias foram obtidas na resolução do mesmo problema ilustrado na Figura 1. Entretanto, na Figura 2, as trajetórias são criadas pelo decremento do parâmetro barreira. Em $\mu=1$, tem-se as estimativas iniciais das magnitudes de tensão - $1,0 \mathrm{pu}$-, em $\mu=0$, tem-se os valores ótimos dessas magnitudes. Como o problema FPO foi resolvido usando-se agora um método diferente, as trajetórias da Figura 2 são distintas daquelas da Figura 1; apenas os valores ótimos das magnitudes das tensões, definidos para $\varepsilon=1$ na Figura 1 , e $\mu=0$ na Figura 2, são os mesmos.

Seja $\mathbf{y}^{0}$ a solução de (17)-(20) para $\mu^{0}$ e $\varepsilon^{0}$. Se um incremento em $\varepsilon^{0}$ ou em $\mu^{0}$ levar a uma variação também incremental no ponto solução tem-se um caso não crítico. Para simplificar a notação define-se $\boldsymbol{\xi}=[\mu, \varepsilon]^{T}$. Tomando uma aproximação linear de $\boldsymbol{\tau}(\mathbf{y}, \boldsymbol{\xi})$ tem-se:

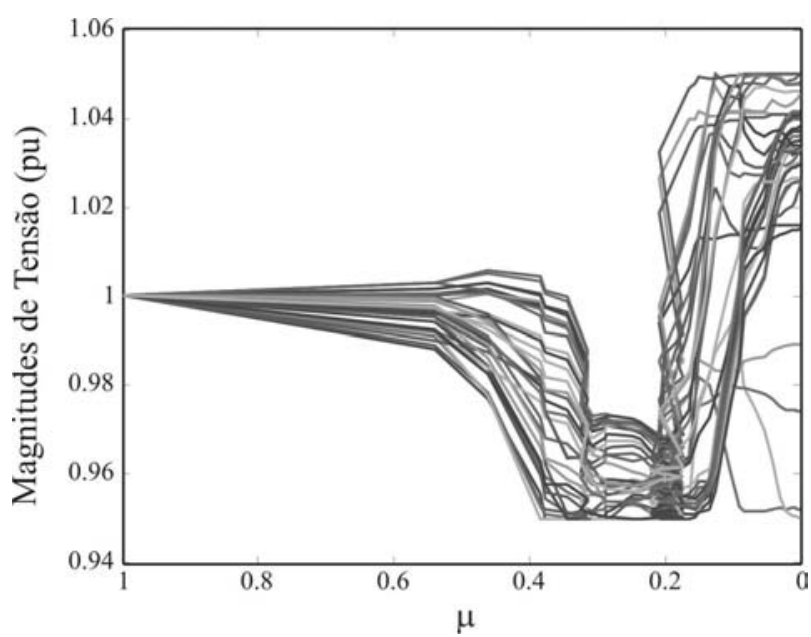

Figura 2: Trajetórias de magnitudes de tensão.

$$
\begin{aligned}
& \boldsymbol{\tau}(\mathbf{y}, \boldsymbol{\xi}) \approx \boldsymbol{\tau}\left(\mathbf{y}^{0}, \boldsymbol{\xi}^{0}\right)+ \\
& {\left[\begin{array}{ll}
\nabla_{y} \boldsymbol{\tau}\left(\mathbf{y}^{0}, \boldsymbol{\xi}^{0}\right) & \nabla_{\xi} \boldsymbol{\tau}\left(\mathbf{y}^{0}, \boldsymbol{\xi}^{0}\right)
\end{array}\right]\left[\begin{array}{c}
\mathbf{y}-\mathbf{y}^{0} \\
\boldsymbol{\xi}-\boldsymbol{\xi}^{0}
\end{array}\right]=\mathbf{0}}
\end{aligned}
$$

Assim, para que o novo ponto obtido após o incremento em $\boldsymbol{\xi}$ satisfaça KKT tem-se que

$$
\mathbf{y}=\mathbf{y}^{0}+\left[\nabla_{y} \boldsymbol{\tau}\left(\mathbf{y}^{0}, \boldsymbol{\xi}^{0}\right)\right]^{-1} \nabla_{\xi} \boldsymbol{\tau}\left(\mathbf{y}^{0}, \boldsymbol{\xi}^{0}\right)\left(\boldsymbol{\xi}-\boldsymbol{\xi}^{0}\right)
$$

Supondo-se que um dos parâmetros do problema seja fixo, o sistema linear anterior possui uma incógnita a mais do que o número de equações. Portanto, sua solução é uma reta. Desta forma, se $\nabla_{y} \boldsymbol{\tau}\left(\mathbf{y}^{0}, \boldsymbol{\xi}^{0}\right)$ é inversível, na vizinhança de $\boldsymbol{\xi}^{0}$, as soluções de KKT estão numa reta que passa por $\left(\mathbf{y}^{0}, \boldsymbol{\xi}^{0}\right)$ constituindo casos não críticos do FPO. Por outro lado, se $\nabla_{y} \boldsymbol{\tau}\left(\mathbf{y}^{0}, \boldsymbol{\xi}^{0}\right)$ é singular, a condição de variação incremental em y não é satisfeita, definindo um caso crítico do problema.

A análise volta-se, portanto, para as situações que levam à singularidade da matriz $\nabla_{y} \boldsymbol{\tau}(\mathbf{y}, \boldsymbol{\xi})$. Uma vez que, em (18), o parâmetro $\mu$ aparece multiplicado por uma constante, essa matriz pode ser escrita como:

$$
\hat{\mathbf{W}}(\mathbf{y}, \varepsilon)=\left[\begin{array}{cc}
\hat{\mathbf{H}}(\mathbf{y}, \varepsilon) & \hat{\mathbf{J}}_{1}^{T}(\mathbf{y}, \varepsilon) \\
\hat{\mathbf{J}}(\mathbf{x}, \varepsilon) & \mathbf{0}
\end{array}\right]
$$

Na equação (23), tem-se

$$
\hat{\mathbf{H}}(\mathbf{y}, \varepsilon)=\left[\begin{array}{cc}
\nabla_{x}^{2} \mathcal{L} & \mathbf{0} \\
\mathbf{0} & \boldsymbol{\Pi}
\end{array}\right],
$$


sendo

$$
\begin{gathered}
\nabla_{x}^{2} \mathcal{L}=\nabla_{x}^{2} f(\mathbf{x}, \varepsilon)+\sum_{k \in K} \lambda_{k} \nabla_{x}^{2} g_{k}(\mathbf{x}, \varepsilon)+\sum_{i \in I} \pi_{i} \nabla_{x}^{2} h_{i}(\mathbf{x}, \varepsilon) \\
\hat{\mathbf{J}}(\mathbf{x}, \varepsilon)=\left[\begin{array}{cc}
\nabla_{x} \mathbf{g}(\mathbf{x}, \varepsilon) & \mathbf{0} \\
\nabla_{x} \mathbf{h}(\mathbf{x}, \varepsilon) & \mathbf{1}
\end{array}\right]
\end{gathered}
$$

e

$$
\begin{aligned}
\hat{\mathbf{J}}_{1}(\mathbf{y}, \varepsilon) & =\left[\begin{array}{cc}
\nabla_{x} \mathbf{g}(\mathbf{x}, \varepsilon) & \mathbf{0} \\
\nabla_{x} \mathbf{h}(\mathbf{x}, \varepsilon) & \mathbf{S}
\end{array}\right] \\
& =\left[\begin{array}{rr}
\nabla_{x} \mathbf{g}(\mathbf{x}, \varepsilon) & \mathbf{0} \\
\nabla_{x} \mathbf{h}(\mathbf{x}, \varepsilon) & \mathbf{1}
\end{array}\right]\left[\begin{array}{ll}
\mathbf{1} & \mathbf{0} \\
\mathbf{0} & \mathbf{S}
\end{array}\right] \\
& =\hat{\mathbf{J}}(\mathbf{x}, \varepsilon) \mathbf{C}
\end{aligned}
$$

onde $\mathbf{S}$ e $\Pi$ matrizes diagonais formadas por $s_{i}$ e $\pi_{i}, i \in I$ e 1 a matriz identidade.

A matriz $\hat{\mathbf{W}}$ é não singular se e somente se: (a) a projeção de $\hat{\mathbf{H}}$ no espaço nulo de $\hat{\mathbf{J}}$ é não singular, (b) $\hat{\mathbf{J}}$ possui posto completo e (c) C é não singular (Guddat et al., 1990). Demonstra-se, no Apêndice, que a condição (a) é satisfeita se e somente se a matriz $\mathbf{H}_{p}$ for não singular. Por outro lado, a função barreira logarítmica assegura que $s_{i}>0, i \in I$. Portanto, a condição (c) é sempre satisfeita. Assim, $\mathbf{W}$ é não singular se e somente se:

(A') A matriz $\hat{\mathbf{J}}$ possui posto completo.

(B’) A matriz $\mathbf{H}_{p}$ é não singular.

Com base nas condições (A') e (B') são definidos dois casos críticos para o problema modificado (Tabela 2). As conseqüências de cada caso crítico são análogas às descritas na Tabela 1 (Guddat et al., 1990). Deve-se notar que o caso crítico tipo 2 pode ocorrer também em situações mais genéricas, nas quais a matriz $\mathbf{H}_{p}$ tem autovalores positivos e negativos antes da criticalidade. Nessas situações, $\hat{\mathbf{W}}$ se torna singular mas não há perda de otimalidade.

Tabela 2: Casos Críticos do Problema Modificado.

\begin{tabular}{|c|l|l|}
\hline Tipo & Violação & Conseqüêencia \\
\hline 1 & Violação de (A'): Para & Perda de factibilidade. Quando \\
& $\begin{array}{l}\boldsymbol{\xi} \rightarrow \boldsymbol{\xi}^{0}, \hat{\mathbf{J}} \text { passa } \\
\text { a ter posto incompleto. }\end{array}$ & $\begin{array}{l}\boldsymbol{\xi} \rightarrow \boldsymbol{\xi}^{0}, \hat{\mathbf{W}} \text { se torna singular. } \\
\text { Além disso, pelo menos um dos multi- } \\
\text { plicadores de Lagrange tende a infinito. O } \\
\text { conjunto factível se torna vazio localmente. }\end{array}$ \\
\hline 2 & $\begin{array}{l}\text { Violação de (B'): Para } \\
\boldsymbol{\xi} \rightarrow \boldsymbol{\xi}^{0}, \text { um autovalor } \\
\text { de } \mathbf{H}_{p} \text { se anula. }\end{array}$ & $\begin{array}{l}\text { Perda de otimalidade. Quando } \\
\boldsymbol{\xi} \rightarrow \boldsymbol{\xi}^{0}, \hat{\mathbf{W}} \text { se torna singular. }\end{array}$ \\
\hline \multicolumn{2}{|l}{} \\
\hline
\end{tabular}

\section{DISCUSSÃO}

Os casos críticos são definidos em pontos específicos $\left(\mathbf{z}^{0}, \varepsilon^{0}\right)$ ou $\left(\mathbf{y}^{0}, \boldsymbol{\xi}^{0}\right)$ que os métodos de otimização encontram durante o processo iterativo. São, portanto, pontos críticos das trajetórias de solução. Observa-se o mau condicionamento das matrizes $\mathbf{W}$ e $\hat{\mathbf{W}}$ na vizinhança de alguns pontos críticos. Tais matrizes aparecem nos sistemas lineares resolvidos a cada iteração dos algoritmos analisados. Conseqüentemente, verifica-se dificuldade de convergência desses algoritmos na vizinhança de tais pontos críticos.

Demonstra-se que, na vizinhança dos pontos críticos 2 e 3 da Tabela 1 e também dos pontos críticos 1 e 2 da Tabela 2 as trajetórias definidas por $\mathbf{z}(\varepsilon)$ ou $\mathbf{y}\left(\boldsymbol{\xi}^{0}\right)$ podem ser aproximadas por parábolas (Guddat et al., 1990). Assim, verifica-se que um pequeno incremento em um parâmetro leva a uma alteração considerável em z ou y. Há, portanto, uma diferença importante entre o comportamento das soluções do problema de fluxo de carga e do problema FPO. Na região vizinha ao limite de factibilidade do fluxo de carga verifica-se uma grande alteração no ponto de operação para um incremento no parâmetro carga. No caso do FPO, esse comportamento é observado na vizinhança do limite de factibilidade ou de um ponto onde um autovalor de $\mathbf{H}_{p}$ se anula para um incremento em um parâmetro, sem que ocorra a infactibilidade. No último caso, são definidas soluções múltiplas para o problema FPO se as condições de otimalidade (i) e (ii) forem satisfeitas e todos os autovalores de $\mathbf{H}_{p}$, menos aquele nulo, forem maiores do que zero.

O estudo mostra que uma análise mais detalhada deve ser feita nas situações onde não ocorre a convergência de programas FPO não lineares. Casos infactíveis são caracterizados por valores elevados dos multiplicadores de Lagrange e pelo posto incompleto de $\mathbf{J}$ ou de $\hat{\mathbf{J}}$. Sendo assim, é importante que, mesmo nos casos em que não houve convergência, os programas FPO forneçam as últimas estimativas obtidas para os multiplicadores de Lagrange. Desta forma, a infactibilidade pode ser detectada e posteriormente tratada, por exemplo, por métodos de corte de carga ou relaxação de restrições. Se existem soluções factíveis, tem-se um caso crítico tipo 2, que pode ser resolvido modificando-se a função objetivo, $f(\cdot)$, para que tenha um mínimo bem definido, ou ainda restringindo-se o conjunto factível do problema através da fixação de algumas de suas variáveis ou redução dos limites impostos nas restrições de desigualdade.

Por fim, deve-se notar que, embora a condição (c) não seja violada devido à barreira logarítmica, essa condição mostra o impacto do parâmetro $\mu$ na convergência do método de pontos interiores. Quanto menor o valor de $\mu$, mais as variáveis de folga das restrições ativas se aproximam de zero, tornando as matrizes $\mathbf{C}$ e $\hat{\mathbf{W}}$ mal condicionadas. Portanto, 
é importante estipular um limite mínimo para $\mu$ durante o procedimento iterativo.

O estudo dos pontos críticos possibilita uma nova visão sobre os problemas de convergência dos programas de fluxo de potência ótimo. Ele tem seus fundamentos na Teoria das $\mathrm{Ca}$ tástrofes, onde se analisam as singularidades de sistemas não lineares (Gilmore, 1981). Problemas de convergência são, em geral, vistos como provenientes de fatores diversos, tais como: má definição das restrições ou variáveis de controle do FPO; infactibilidade; uso de uma função objetivo difícil; escolha errônea de escalares utilizados nos algoritmos, por exemplo, tamanho do passo na atualização das variáveis, fatores de ponderação, tolerância para convergência; etc.. Este estudo entende que todos esses fatores, em última análise, levam os algoritmos a estimativas de solução, ou ainda, a regiões do espaço de otimização, problemáticas, que afetam o condicionamento da matriz do método de Newton. Tais regiões são vizinhas aos, ou ainda, os próprios pontos críticos. Portanto, na ótica da Teoria das Catástrofes, não há diferença entre os problemas numéricos provenientes dos métodos de resolução e os pontos críticos; os problemas numéricos são causados pelos pontos críticos. É importante observar que tal afirmativa não é simplista, pois a aplicação do conceito de pontos críticos em metodologias para aumentar a robustez dos algoritmos de FPO não é trivial. Em primeiro lugar, os pontos críticos são definidos localmente. Portanto, o encontro de tais pontos durante o processo iterativo não significa a inexistência mínimo bem definido para o problema. Em segundo lugar, embora possamos classificar as singularidades em dois tipos apenas, o fatores que levam a elas são inúmeros e, portanto, evitá-las é difícil. Por fim, a quantificação de alguns conceitos usados anteriormente não é simples. Por exemplo, os números de condicionamento das matrizes $\mathbf{W}$ e $\hat{\mathbf{W}}$ dependem das características dos sistemas analisados, ou seja, do nível de potência reativa, parâmetros de linha, etc. Um determinado número de condicionamento pode ser razoável para um dado sistema e muito ruim para outro, causando problemas de convergência. Portanto, o uso eficiente dos conceitos anteriores para aumentar a robustez de programas de FPO para diferentes sistemas e condições operativas é um desafio.

\section{EXEMPLOS DE CASOS CRÍTICOS}

A seguir, são analisados exemplos de casos críticos definidos para o problema modificado. O algoritmo utilizado para acompanhar as trajetórias ótimas é descrito em (Almeida and Salgado, 2000). Esse trabalho apresenta um método de acompanhamento das soluções do FPO onde o perâmetro $\varepsilon$ é incrementado no Passo preditor e o parâmetro $\mu$ é decrementado no Passo Corretor, baseado no método primal-dual de pontos interiores. Sendo assim, a existência de casos críticos para variações nos dois parâmetros pode ser avaliada.

Os casos críticos foram obtidos com um equivalente da Região Sul com 350 barras e carga de 4405MW e 1158MVAr. O comportamento de $\mathbf{J}$ é analisado através de seu mínimo valor singular. Quando esse valor se aproxima de zero, $\hat{\mathbf{J}}$ passa a ter posto incompleto.

\subsection{Caso Crítico Tipo 1}

Analisa-se primeiramente o caso crítico através de alterações no parâmetro $\varepsilon$, que modifica as cargas ativas e reativas do sistema. Na Tabela 3, em $\varepsilon=0$, tem-se a condição inicial de carregamento do sistema; em $\varepsilon=1,31$ tem-se o limite de máximo carregamento. A Tabela mostra , para os dois valores de $\varepsilon$, os máximos valores absolutos dos multiplicadores de Lagrange das restrições de desigualdade, $\max \left|\pi_{i}\right|$, os menores autovalores de $\mathbf{H}_{p}$, os mínimos valores singulares de $\hat{\mathbf{J}}$ e os números de condicionamento de $\hat{\mathbf{W}}$. Observa-se que $\max \left|\pi_{i}\right|$ se torna elevado, o mínimo valor singular de $\hat{\mathbf{J}}$ se aproxima de zero e o condicionamento de $\hat{\mathbf{W}}$ se deteriora com o aumento da carga. Verifica-se também que o menor autovalor de $\mathbf{H}_{p}$ se mantém mais ou menos constante. Tal comportamento caracteriza o caso crítico tipo 1 , que define o limite de factibilidade do FPO.

A Figura 3 mostra algumas trajetórias de magnitudes de tensão de barra que correspondem às soluções do FPO definidas para valores crescentes de $\varepsilon$. Nota-se que essas magnitudes apresentam uma grande variação quando $\varepsilon$ se aproxima de 1,31 , configurando uma situação análoga à observada no limite de factibilidade do fluxo de carga. A Figura 4 mostra as trajetórias dos multiplicadores de Lagrange associados à limites de tensão na vizinhança do ponto crítico. Nota-se que os multiplicadores apresentam uma variação abrupta na vizinhança do ponto crítico.

O comportamento descrito anteriormente pode ser observado também quando se busca resolver o problema para $\varepsilon$ fixo em 1,33 - ou seja, acima do limite máximo de carregamento pelo método de pontos interiores. A Figura 5 mostra, para este caso, as trajetórias de estimativas das magnitudes de tensão em função de $\mu$. Não é possível reduzir o parâmetro $\mu$ além de $0,005 \mathrm{e}$, para esse valor, $\max \left|\pi_{i}\right|=2,17 \times 10^{6}$, o mínimo valor singular de $\widehat{\mathbf{J}}$ é $3,96 \times 10^{-14}$, o número de

Tabela 3: Indicadores - Caso Crítico Tipo 1.

\begin{tabular}{|c|c|c|c|c|}
\hline Parâmetro & $\begin{array}{c}\text { Máximo } \\
\left|\pi_{i}\right|, i \in A\end{array}$ & $\begin{array}{c}\text { Min. Valor } \\
\text { Sing. de } \hat{\mathbf{J}}\end{array}$ & $\begin{array}{c}\text { Min. Autov. } \\
\text { de } \mathbf{H}_{p}\end{array}$ & $\begin{array}{c}\text { Num. Cond. } \\
\text { de } \hat{\mathbf{W}}\end{array}$ \\
\hline$\varepsilon=0$ & 0,8518 & 0,099 & 0,1398 & $2,36 \times 10^{9}$ \\
\hline$\varepsilon=1,31$ & $1,17 \times 10^{4}$ & $4,04 \times 10^{-14}$ & 0,3698 & $7,31 \times 10^{18}$ \\
\hline
\end{tabular}




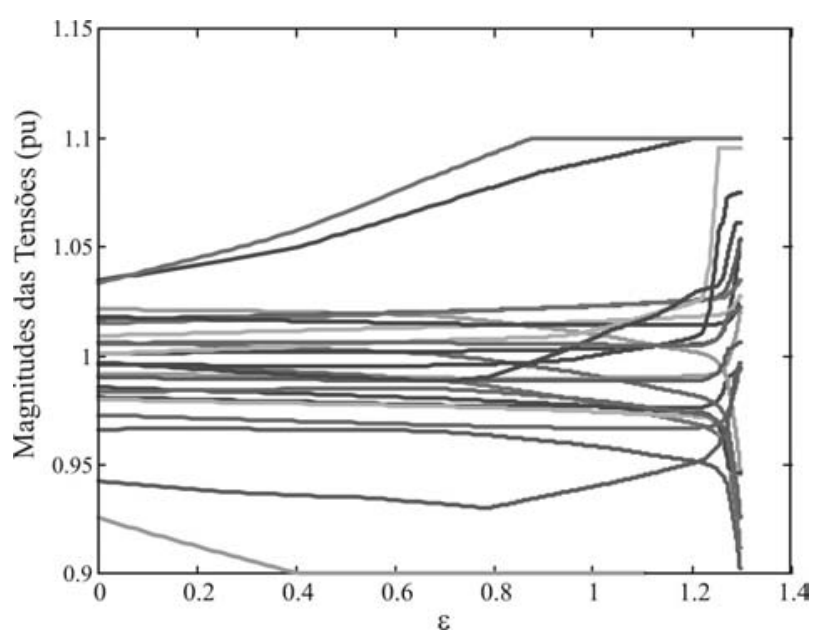

Figura 3: Magnitudes de Tensão - Caso Crítico 1.

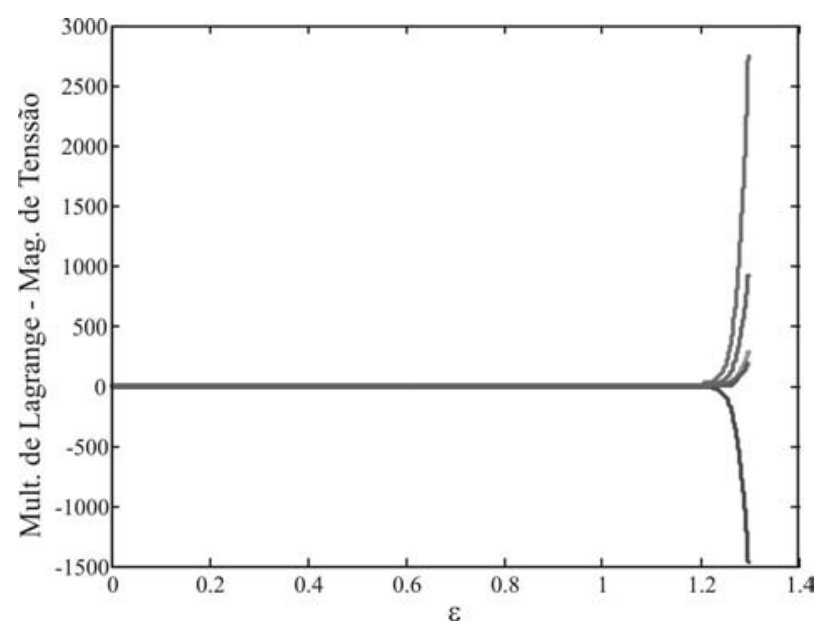

Figura 4: Multiplicadores de Lagrange - Caso Crítico 1.

condicionamento de $\hat{\mathbf{W}}$ é $5,64 \times 10^{23}$ e a estimativa de solução obtida pelo algoritmo de pontos interiores não satisfaz às restrições impostas, configurando um caso infactível.

\subsection{Caso Crítico Tipo 2}

A Tabela 4 e as Figuras 6 e 7 ilustram um caso crítico do tipo 2 , definido pelo decremento do parâmetro $\mu$ no método primal-dual de pontos interiores. Neste caso, $\varepsilon$ é fixo. A tabela mostra, para os pontos inicial $(\mu=1)$ e final $\left(\mu=1 \times 10^{-10}\right)$ do processo iterativo, os máximos valores absolutos dos multiplicadores de Lagrange das restrições de desigualdade, $\max \left|\pi_{i}\right|$, os mínimos valores singulares de $\hat{\mathbf{J}}$ os mínimos autovalores de $\mathbf{H}_{p}$ e os números de condicionamento de $\hat{\mathbf{W}}$. Observa-se que, quando $\mu \rightarrow 0$, o menor

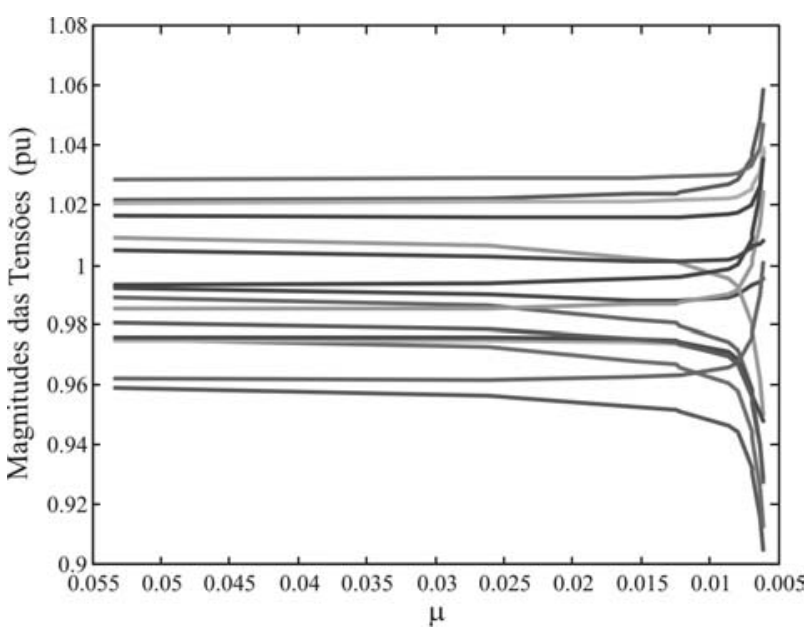

Figura 5: Mag. Tensão versus $\mu$ - Caso Crítico 1.

autovalor de $\mathbf{H}_{p}$ se aproxima de zero e o condicionamento de $\hat{\mathbf{W}}$ se deteriora. Por outro lado, $\max \left|\pi_{i}\right|$ se mantém pequeno e o mínimo valor singular de $\hat{\mathbf{J}}$ quase não se altera, indicando que não se trata de um caso infactível do FPO.

As Figuras 6 e 7 mostram o comportamento das magnitudes das tensões de barra e de taps de transformadores na vizinhança do ponto crítico definido para $\mu=1 \times 10^{-10}$. Nota-se que, quando $\mu \rightarrow 0$, essas variáveis experimentam uma grande alteração. Isso confirma o fato que, no ponto crítico, as trajetórias das soluções podem ser aproximadas por parábolas.

Este caso foi obtido na tentativa de minimizar o desvio quadrático das tensões em relação a 1, $0 p u$. No final do processo iterativo, $\mathbf{H}_{p}$ possui um autovalor nulo e os demais autovalores maiores do que zero, indicando que o problema admite múltiplas soluções. Neste caso, o algoritmo de pontos interiores convergiu após 74 iterações, o que é aproximadamente três vezes o número usual gasto em testes com o sistema de 350 barras.

Tabela 4: Indicadores - Caso Crítico Tipo 2.

\begin{tabular}{|c|c|c|c|c|}
\hline Parâmetro & $\begin{array}{c}\text { Máximo } \\
\left|\pi_{i}\right|, i \in A\end{array}$ & $\begin{array}{c}\text { Min. Valor } \\
\text { Sing. de } \hat{\mathbf{J}}\end{array}$ & $\begin{array}{c}\text { Min. Autov. } \\
\text { de } \mathbf{H}_{p}\end{array}$ & $\begin{array}{c}\text { Num. Cond. } \\
\text { de } \hat{\mathbf{W}}\end{array}$ \\
\hline$\mu=1$ & 0,0 & 0,1120 & 29,56 & $3,28 \times 10^{9}$ \\
\hline$\mu=1 \times 10^{-10}$ & 0,2169 & 0,0990 & $9,32 \times 10^{-6}$ & $1,13 \times 10^{14}$ \\
\hline
\end{tabular}




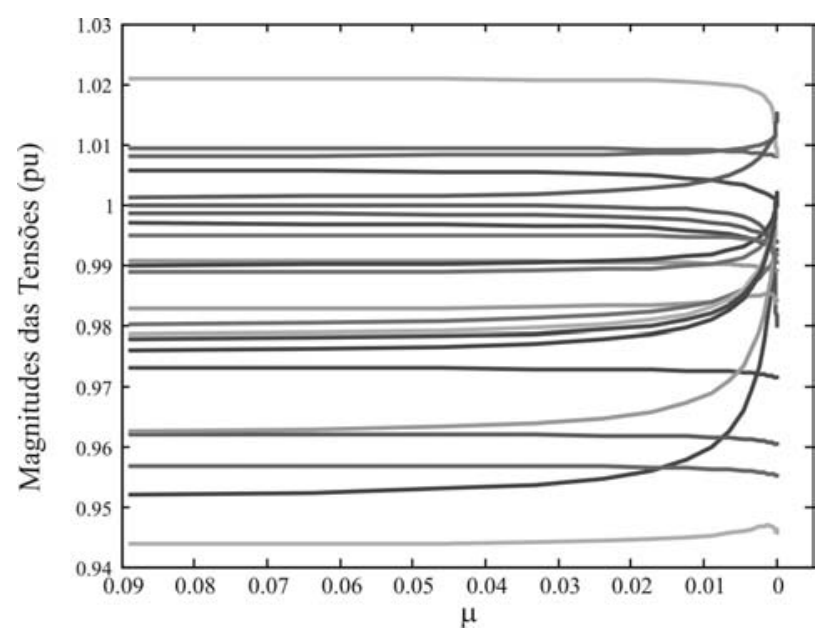

Figura 6: Magnitudes de Tensão - Caso Crítico 2.

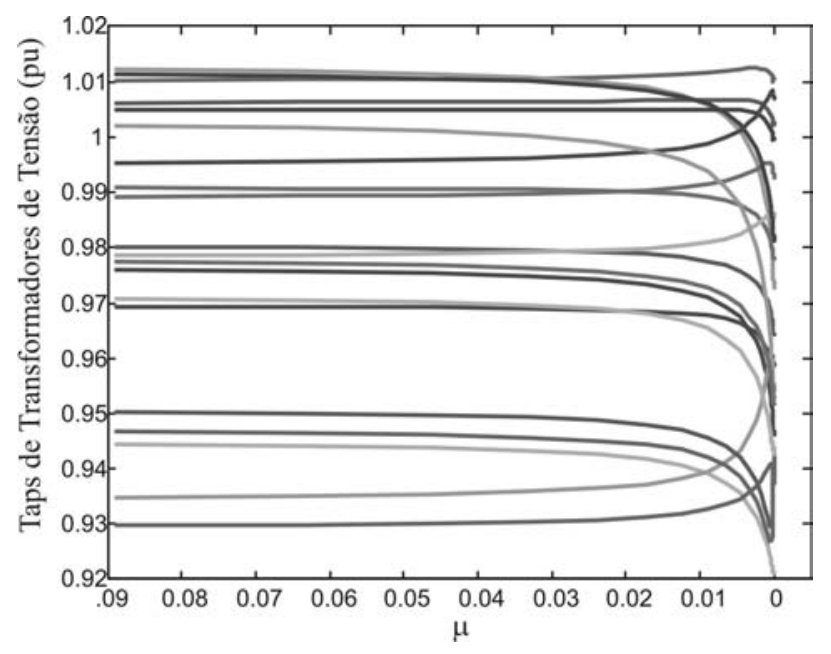

Figura 7: Taps de Transformadores - Caso Crítico 2.

\section{CONCLUSÕES}

O estudo mostra que há meios eficientes para se verificar as causas genéricas para a divergência de algoritmos de FPO baseados no método de Newton ou no método primal-dual de pontos interiores. Infelizmente, tais meios são baseados em técnicas matemáticas pouco usuais em pacotes comerciais de FPO: métodos para calcular a Hessiana projetada e seus autovalores ou os valores singulares do Jacobiano das restrições do problema. A diferenciação entre casos infactíveis e aqueles para os quais existe solução é de grande valia para os usuários dos programas FPO. Os resultados podem ser empregados para aumentar a robustez desses programas computacionais. Entretanto, somente a diferenciação dos casos críticos é insuficiente. É necessário também desenvolver mé- todos eficientes para resolver esses casos. Como diferentes situações podem levar aos casos críticos, a quantificação de alguns índices empregados no estudo é complicada e, além disso, as relações matemáticas que definem os pontos críticos são complexas, tal resolução nem sempre é trivial.

\section{APÊNDICE}

Seja $\mathbf{u}$ um vetor do espaço nulo de $\hat{\mathbf{J}}$ e $\mathbf{v}_{1}, \mathbf{v}_{2}, \ldots, \mathbf{v}_{r}$ uma base para o espaço nulo de $\mathbf{J}_{+}$. Podemos escrever:

$$
\begin{gathered}
\hat{\mathbf{J}} \mathbf{u}=\left[\begin{array}{ccc}
\nabla_{x} \mathbf{g}(\mathbf{x}, \varepsilon) & \mathbf{0} & \mathbf{0} \\
\nabla_{x} \mathbf{h}_{A^{+}}(\mathbf{x}, \varepsilon) & \mathbf{1}_{A^{+}} & \mathbf{0} \\
\nabla_{x} \mathbf{h}_{N}(\mathbf{x}, \varepsilon) & \mathbf{0} & \mathbf{1}_{N}
\end{array}\right]\left[\begin{array}{l}
\mathbf{u}_{1} \\
\mathbf{u}_{2} \\
\mathbf{u}_{3}
\end{array}\right]=\mathbf{0} \\
\mathbf{J}_{+} \mathbf{v}_{i}=\left[\begin{array}{c}
\nabla_{x} \mathbf{g}(\mathbf{x}, \varepsilon) \\
\nabla_{x} \mathbf{h}_{A^{+}}(\mathbf{x}, \varepsilon)
\end{array}\right] \mathbf{v}_{i}=\mathbf{0}, i=1, \ldots, r
\end{gathered}
$$

sendo $A^{+}$o conjunto das restrições de desigualdade ativas associadas a $\pi_{i}>0, N$ o conjunto das demais restrições de desigualdade, $\mathbf{1}_{A^{+}}$e $\mathbf{1}_{N}$ matrizes identidade com dimensões iguais aos números de componentes de $A^{+}$e $N$, respectivamente, e $\mathbf{h}_{A^{+}}$o vetor composto por $h_{i}, i \in A^{+}$.

Tendo em vista as equações anteriores, para $\kappa_{i}, i=1, \ldots, r$ constantes, $\mathbf{u}_{1}, \mathbf{u}_{2}$ e $\mathbf{u}_{3}$ podem ser expressos como

$$
\begin{aligned}
& \mathbf{u}_{1}=\sum_{i=1}^{r} \kappa_{i} \mathbf{v}_{i}, \\
& \mathbf{u}_{2}=-\nabla_{x} \mathbf{h}_{A^{+}}(\mathbf{x}, \varepsilon)\left(\sum_{i=1}^{r} \kappa_{i} \mathbf{v}_{i}\right)=\mathbf{0}, \\
& \mathbf{u}_{3}=-\nabla_{x} \mathbf{h}_{N}(\mathbf{x}, \varepsilon)\left(\sum_{i=1}^{r} \kappa_{i} \mathbf{v}_{i}\right) .
\end{aligned}
$$

Portanto, $\mathbf{u}_{1}$ pertence ao espaço nulo de $\mathbf{J}_{+}$.

Seja U uma matriz cujas colunas são os vetores que formam uma base para o espaço nulo de $\hat{\mathbf{J}}$. Podemos representá-la como

$$
\mathbf{U}=\left[\begin{array}{l}
\mathbf{U}_{1} \\
\mathbf{U}_{2} \\
\mathbf{U}_{3}
\end{array}\right]
$$

onde $\mathbf{U}_{1}, \mathbf{U}_{2}$ e $\mathbf{U}_{3}$ são matrizes cujas colunas são formadas pelos vetores $\mathbf{u}_{1}, \mathbf{u}_{2}$ e $\mathbf{u}_{3}$, respectivamente.

Para analisar a projeção de $\hat{\mathbf{H}}$ no espaço nulo de $\hat{\mathbf{J}}$ toma-se

$$
\begin{aligned}
\hat{\mathbf{H}}_{p}= & \mathbf{U}^{T} \hat{\mathbf{H}} \mathbf{U}= \\
& {\left[\begin{array}{lll}
\mathbf{U}_{1}^{T} & \mathbf{U}_{2}^{T} & \mathbf{U}_{3}^{T}
\end{array}\right]\left[\begin{array}{ccc}
\nabla_{x}^{2} \mathcal{L} & \mathbf{0} & \mathbf{0} \\
\mathbf{0} & \boldsymbol{\Pi}_{A^{+}} & \mathbf{0} \\
\mathbf{0} & \mathbf{0} & \boldsymbol{\Pi}_{N}
\end{array}\right]\left[\begin{array}{c}
\mathbf{U}_{1} \\
\mathbf{U}_{2} \\
\mathbf{U}_{3}
\end{array}\right], }
\end{aligned}
$$


ou ainda,

$$
\hat{\mathbf{H}}_{p}=\mathbf{U}_{1}^{T} \nabla_{x}^{2} \mathcal{L} \mathbf{U}_{1}+\mathbf{U}_{2}^{T} \boldsymbol{\Pi}_{A^{+}} \mathbf{U}_{2}+\mathbf{U}_{3}^{T} \boldsymbol{\Pi}_{N} \mathbf{U}_{3},
$$

sendo $\Pi_{A+}$ e $\Pi_{N}$ matrizes diagonais formadas por $\pi_{i}>0 \mathrm{e}$ $\pi_{i}=0$, respectivamente.

Uma vez que, na equação (25), $\pi_{i}=0, i \notin A, \nabla_{x}^{2} \mathcal{L}$ pode ser reescrita como

$$
\nabla_{x}^{2} \mathcal{L}=\nabla_{x}^{2} f(\cdot)+\sum_{k \in K} \lambda_{k} \nabla_{x}^{2} g_{k}(\cdot)+\sum_{i \in A} \pi_{i} \nabla_{x}^{2} h_{i}(\cdot)=\mathbf{H} .
$$

Substituindo (34) em (33), já que $\boldsymbol{\Pi}_{N}=0$ e, de (30), $\mathbf{U}_{2}=$ $\mathbf{0}$, tem-se

$$
\hat{\mathbf{H}}_{p}=\mathbf{U}_{1}^{T} \mathbf{H U}_{1} .
$$

Uma vez que as colunas de $\mathbf{U}_{1}$, pertencem ao espaço nulo de $\mathbf{J}_{+}$, a projeção de $\hat{\mathbf{H}}$ no espaço nulo de $\hat{\mathbf{J}}$ - matriz $\hat{\mathbf{H}}_{p}$ - é singular se e somente se a projeção de $\mathbf{H}$ no espaço nulo de $\mathbf{J}_{+}$- matriz $\mathbf{H}_{p}$ - também for.

\section{AGRADECIMENTOS}

Este trabalho foi feito com o apoio da CAPES e do CNPq.

\section{REFERÊNCIAS}

Alaç, O., Prais, M. and Stott, B. (1990). Further developments in lp-based optimal power flow, IEEE Transactions on Power Systems 15(3): 697-711.

Almeida, K. C. (1998). Metodologia de Análise do Comportamento das Soluções do Fluxo de Potência Ótimo sob Carga Variável, Departamento de Engenharia Elétrica UFSC. Monografia, Concurso para Professor Adjunto.

Almeida, K. C. and Galiana, F. D. (1996). Critical cases in the optimal power flow, IEEE Transactions on Power Systems 11(3): 1509-1518.

Almeida, K. C., Galiana, F. D. and Soares, S. (1994). A general parametric optimal power flow, IEEE Transactions on Power Systems 9(1): 540-547.

Almeida, K. C. and Salgado, R. S. (2000). Optimal power flow solutions under variable load conditions, IEEE Transactions on Power Systems 15(4): 1204-1211.

Barboza, L. V. and Salgado, R. (2001). Restoring solutions for unsolvable cases via minimum load shedding for a specific direction, Proceedings of the Power Industry Computer Applications Conference, Vol. I, Sydney - Australia, pp. 1-7.

Fang, S. C. and Puthempura, S. (1993). Linear Optimization and Extensions, Prentice-Hall.
Gilmore, R. (1981). Catastrophe Theory for Scientists and Engineers, Dover Publications.

Granville, S., Mello, J. C. and Melo, A. C. G. (1996). Application of interior point methods to power flow unsolvability, IEEE Transactions on Power Systems 11(2): 1096-1103.

Guddat, J., Guerra Vazquez, F. and Jongen, H. T. (1990). Parametric Optimization: Singularities, Pathfollowing and Jumps, John Wiley and Sons.

Luenberger, D. (1984). Linear and Nonlinear Optimization Second Edition, Addison Wesley.

Monticelli, A. and Liu, W. H. E. (1992). Adaptative movement penalty function for newton optimal power flow, IEEE Transactions on Power Systems 7(1): 334-342.

Moyano, C. F. (2003). Soluções de Máximo Carregamento no Contexto da Teoria da Bifurcação Sela-Nó, Departamento de Engenharia Elétrica - UFSC. Monografia, Exame de Qualificação de Doutorado.

Oliveira, E. J., Moreira, W. C., Pereira, J. L. and Willer, L. (2003). Solução de sistemas de potência mal condicionados usando a técnica de relaxamento de restrições, Anais do XVII SNPTEE, Vol. I, Uberlândia, pp. 1-6.

Sun, D. I., Ashley, B., Brewer, B., Hughes, A. and Tinney, W. F. (1984). Optimal power flow by newton method, IEEE Transactions on Power Apparatus and Systems 103(10): 2864-2875.

Sun, D. I., Hu, T. I., Lin, C. J. and Chen, C. M. (1988). Experiences with implementing optimal power flow for reactive rescheduling in the taiwan power system, IEEE Transactions on Power Systems 3(3): 1193-1120. 